The third type bunch of connections induced by an analog of Norden's normalization for the Grassmann-like manifold of centered planes

\author{
Olga Belova
}




\title{
THE THIRD TYPE BUNCH OF CONNECTIONS INDUCED BY AN ANALOG OF NORDEN'S NORMALIZATION FOR THE GRASSMANN-LIKE MANIFOLD OF CENTERED PLANES
}

\author{
OLGA BELOVA
}

\begin{abstract}
The Grassmann-like manifold of centered m-planes (i.e. passing through a fixed point) and the principal bundle over it are considered in $n$-dimensional projective space. An analogy of Norden's construction of strong normalization induces bunches of connections of three types in the fibering associated to the Grassmann-like manifold which are related to each other. The third type bunch of group connections is constructed and a unique connection from this bunch is distinguished.
\end{abstract}

2000 Mathematics Subject Classification: Primary 53A20, 53B25; Secondary 53B15

Keywords: projective space, Grassmann-like manifold of centered planes, Norden's normalization, object of group connection, average connection, bunch of connections

In the $n$-dimensional projective space $P_{n}$, we consider the moving frame $\left\{A, A_{I}\right\}$ $(I, \ldots=\overline{1, n})$ with the derivation formulas

$$
\begin{aligned}
d A & =\theta A+\omega^{I} A_{I}, \\
d A_{I} & =\theta A_{I}+\omega_{I}^{J} A_{J}+\omega_{I} A,
\end{aligned}
$$

where the form $\theta$ plays the role of a proportionality factor and the structure forms $\omega^{I}, \omega_{J}^{I}, \omega_{I}$ of the projective group $\operatorname{GP}(n)$, which acts effectively on $P_{n}$, satisfy the Cartan equations

$$
\begin{aligned}
& D \omega^{I}=\omega^{J} \wedge \omega_{J}^{I}, \\
& D \omega_{J}^{I}=\omega_{J}^{K} \wedge \omega_{K}^{I}+\delta_{J}^{I} \omega_{K} \wedge \omega^{K}+\omega_{J} \wedge \omega^{I}, \\
& D \omega_{I}=\omega_{I}^{J} \wedge \omega_{J} .
\end{aligned}
$$

We consider the Grassmann-like manifold $\mathrm{Gr}^{*}(m, n)$ [3] of centered $m$-dimensional planes. This manifold is given by the equations

$$
\omega^{a}=\Lambda_{\alpha}^{a} \omega^{\alpha}+\Lambda_{\alpha}^{a b} \omega_{b}^{\alpha} \quad(a, \ldots=\overline{1, m} ; \quad \alpha, \ldots=\overline{m+1, n}),
$$


where the components of the first order fundamental object $\Lambda=\left\{\Lambda_{\alpha}^{a}, \Lambda_{\alpha}^{a b}\right\}$ satisfy the differential equations

$$
\begin{aligned}
\Delta \Lambda_{\alpha}^{a}+\Lambda_{\alpha}^{a b} \omega_{b}+\omega_{\alpha}^{a} & =\Lambda_{\alpha \beta}^{a} \omega^{\beta}+\Lambda_{\alpha \beta}^{a b} \omega_{b}^{\beta}, \\
\Delta \Lambda_{\alpha}^{a b} & =\bar{\Lambda}_{\alpha \beta}^{a b} \omega^{\beta}+\Lambda_{\alpha \beta}^{a b c} \omega_{c}^{\beta} .
\end{aligned}
$$

The principal fiber bundle $G^{*}\left(\operatorname{Gr}^{*}(m, n)\right)$ with the structure equations

$$
\begin{aligned}
D \omega^{\alpha}= & \omega^{\beta} \wedge \omega_{\beta}^{\alpha}+\Lambda_{\beta}^{a} \omega^{\beta} \wedge \omega_{a}^{\alpha}+\Lambda_{\beta}^{a b} \omega_{b}^{\beta} \wedge \omega_{a}^{\alpha}, \\
D \omega_{a}^{\alpha}= & \omega_{a}^{b} \wedge \omega_{b}^{\alpha}+\omega_{a}^{\beta} \wedge \omega_{\beta}^{\alpha}+\omega_{a} \wedge \omega^{\alpha} \\
D \omega_{b}^{a}= & \omega_{b}^{c} \wedge \omega_{c}^{a}+\left(\delta_{b}^{a} \Lambda_{\alpha}^{c} \omega_{c}+\delta_{b}^{a} \omega_{\alpha}+\Lambda_{\alpha}^{a} \omega_{b}\right) \wedge \omega^{\alpha} \\
& +\left(\delta_{b}^{a} \Lambda_{\alpha}^{e c} \omega_{e}-\delta_{b}^{c} \omega_{\alpha}^{a}+\Lambda_{\alpha}^{a c} \omega_{b}\right) \wedge \omega_{c}^{\alpha}, \\
D \omega_{\beta}^{\alpha}= & \omega_{\beta}^{\gamma} \wedge \omega_{\gamma}^{\alpha}+\left(\delta_{\beta}^{\alpha} \Lambda_{\gamma}^{a} \omega_{a}+\delta_{\beta}^{\alpha} \omega_{\gamma}+\delta_{\gamma}^{\alpha} \omega_{\beta}\right) \wedge \omega^{\gamma}+\left(\delta_{\beta}^{\alpha} \Lambda_{\gamma}^{b a} \omega_{b}+\delta_{\gamma}^{\alpha} \omega_{\beta}^{a}\right) \wedge \omega_{a}^{\gamma}, \\
D \omega_{\alpha}^{a}= & \omega_{\alpha}^{b} \wedge \omega_{b}^{a}+\omega_{\alpha}^{\beta} \wedge \omega_{\beta}^{a}+\left(\Lambda_{\beta}^{a} \omega_{\alpha}\right) \wedge \omega^{\beta}+\left(\Lambda_{\beta}^{a b} \omega_{\alpha}\right) \wedge \omega_{b}^{\beta}, \\
D \omega_{a}= & \omega_{a}^{b} \wedge \omega_{b}+\omega_{a}^{\alpha} \wedge \omega_{\alpha}, \\
D \omega_{\alpha}= & \omega_{\alpha}^{a} \wedge \omega_{a}+\omega_{\alpha}^{\beta} \wedge \omega_{\beta}
\end{aligned}
$$

is constructed over the manifold $\mathrm{Gr}^{*}(m, n)$, the stationarity subgroup $G^{*}$ of the centered plane $L_{m}^{*}$ is the typical fiber. In the principal fiber bundle the connection is given in G. F. Laptev's [3] method by the field of the connection object $\Gamma$. This object contains the subobject

$$
\Gamma_{1}=\left\{\Gamma_{b \alpha}^{a}, L_{b \alpha}^{a c}, \Gamma_{\beta \gamma}^{\alpha}, L_{\beta \gamma}^{\alpha a}\right\} .
$$

An analog of strong Norden's normalization for this manifold is carried out. It consists of the fields of the planes $C_{n-m-1}$ and $N_{m-1}$ :

$$
L_{m}^{*} \cap C_{n-m-1}=\varnothing, \quad A \notin N_{m-1} \subset L_{m}^{*} .
$$

The planes $C_{n-m-1}$ and $N_{m-1}$ will be set by the points $B_{\alpha}=A_{\alpha}+\lambda_{\alpha}^{a} A_{a}+\lambda_{\alpha} A$ and $B_{a}=A_{a}+\lambda_{a} A$, correspondingly.

The differential equations for the components of the clothing quasitensor $\lambda=$ $\left\{\lambda_{\alpha}^{a}, \lambda_{\alpha}, \lambda_{a}\right\}$ have the form

$$
\begin{aligned}
\Delta \lambda_{\alpha}^{a}+\omega_{\alpha}^{a} & =\lambda_{\alpha \beta}^{a} \omega^{\beta}+\lambda_{\alpha \beta}^{a b} \omega_{b}^{\beta}, \\
\Delta \lambda_{\alpha}+\lambda_{\alpha}^{a} \omega_{a}+\omega_{\alpha} & =\lambda_{\alpha \beta} \omega^{\beta}+\chi_{\alpha \beta}^{a} \omega_{a}^{\beta}, \\
\Delta \lambda_{a}+\omega_{a} & =\lambda_{a \alpha} \omega^{\alpha}+\lambda_{a \alpha}^{b} \omega_{b}^{\alpha} .
\end{aligned}
$$


This normalization induces the connections of three types $[1,3] \stackrel{01}{\Gamma}, \stackrel{02}{\Gamma}, \stackrel{03}{\Gamma}$ in the fibering associated with the manifold $\mathrm{Gr}^{*}(m, n)$. Moreover,

$$
\stackrel{01}{\Gamma}=\frac{1}{2}(\stackrel{02}{\Gamma}+\stackrel{03}{\Gamma})
$$

Thus the first type connection is average [4] with respect to the connections of the remaining types.

In [2] it is proved that this clothing of the manifold $\operatorname{Gr}^{*}(m, n)$ induces the first type bunch of connections in the fibering $G^{*}\left(\operatorname{Gr}^{*}(m, n)\right)$. From this bunch a unique first type connection is distinguished. In [1], because the covariant derivatives are tensors, we construct the second type bunch of connections. A unique second type connection is distinguished from this bunch.

These bunches of connections $[1,2]$ have identical parameters. These parameters are the components of the subobject $\Gamma_{1}$. In consideration of the correlation between the group connections of three types, we can construct the third type bunch of connections with the object

$$
\stackrel{3}{\Gamma}=2 \stackrel{1}{\Gamma}-\stackrel{2}{\Gamma} \text {. }
$$

The dependent components of this bunch are

$$
\begin{aligned}
& \stackrel{3}{\Gamma}_{\alpha \beta}^{a}=\lambda_{\gamma}^{a} \Gamma_{\alpha \beta}^{\gamma}-\lambda_{\alpha}^{b} \Gamma_{b \beta}^{a}-\lambda_{\alpha \beta}^{a}+2 \lambda_{\alpha} \mu_{\beta}^{a}, \\
& \stackrel{3}{L}_{\alpha \beta}^{a b}=\lambda_{\gamma}^{a} L_{\alpha \beta}^{\gamma b}-\lambda_{\alpha}^{c} L_{c \beta}^{a b}-\lambda_{\alpha \beta}^{a b}+2 \lambda_{\alpha}^{b} \lambda_{\beta}^{a}-2 \lambda_{\alpha} \Lambda_{\beta}^{a b}, \\
& \stackrel{3}{L}_{a \alpha}=\lambda_{b} \Gamma_{a \alpha}^{b}+2 \lambda_{a} \lambda_{b} \Lambda_{\alpha}^{b}+2 \lambda_{a} \eta_{\alpha}-\lambda_{a \alpha}, \\
& \stackrel{3}{\Pi}_{a \alpha}^{b}=\lambda_{c} L_{a \alpha}^{c b}+2 \lambda_{a} \lambda_{c} \Lambda_{\alpha}^{c b}+2 \delta_{a}^{b} \eta_{\alpha}-\lambda_{a \alpha}^{b}, \\
& \stackrel{3}{L}_{\alpha \beta}^{b}=\lambda_{\gamma} \Gamma_{\alpha \beta}^{\gamma}-\lambda_{\alpha}^{a} \lambda_{b} \Gamma_{a \beta}^{b}+2 \lambda_{\alpha} \lambda_{\beta}-\lambda_{\alpha \beta}+\lambda_{\alpha}^{a} \lambda_{a \beta}-2 \lambda_{\alpha}^{a} \lambda_{a} \eta_{\beta}-2 \lambda_{\alpha}^{a} \Lambda_{\beta}^{b} \lambda_{a} \lambda_{b}, \\
& \stackrel{3}{\Pi}_{\alpha \beta}^{a}=\lambda_{\gamma} L_{\alpha \beta}^{\gamma a}-\lambda_{\alpha}^{b} \lambda_{c} L_{b \beta}^{c a}-2 \lambda_{\alpha}^{b} \lambda_{b} \lambda_{c} \Lambda_{\beta}^{c a}+2 \lambda_{\alpha}^{a} \lambda_{b} \lambda_{\beta}^{b}-\chi_{\alpha \beta}^{a}+\lambda_{\alpha}^{b} \lambda_{b \beta}^{a},
\end{aligned}
$$

where

$$
\begin{aligned}
\mu_{\alpha}^{a} & =\lambda_{\alpha}^{a}-\Lambda_{\alpha}^{a}, \\
\eta_{\alpha} & =\lambda_{\alpha}-\lambda_{\alpha}^{a} \lambda_{a} .
\end{aligned}
$$

Taking into account the covered subobject $\stackrel{0}{\Gamma_{1}}$ [3]

$$
\begin{array}{ll}
\stackrel{0}{\Gamma}_{b \alpha}^{a}=-\delta_{b}^{a} \lambda_{\alpha}+\mu_{\alpha}^{a} \lambda_{b}+\delta_{b}^{a} \mu_{\alpha}^{c} \lambda_{c}, & \stackrel{0}{L}_{b \alpha}^{a c}=\delta_{b}^{c} \lambda_{\alpha}^{a}-\left(\delta_{b}^{a} \Lambda_{\alpha}^{e c}+\delta_{b}^{e} \Lambda_{\alpha}^{a c}\right) \lambda_{e}, \\
\stackrel{0}{\Gamma}_{\beta \gamma}^{\alpha}=-\delta_{\gamma}^{\alpha} \lambda_{\beta}-\delta_{\beta}^{\alpha} \lambda_{\gamma}+\delta_{\beta}^{\alpha} \mu_{\gamma}^{a} \lambda_{a}, & { }^{0} L_{\beta \gamma}^{\alpha a}=-\delta_{\gamma}^{\alpha} \lambda_{\beta}^{a}-\delta_{\beta}^{\alpha} \Lambda_{\gamma}^{b a} \lambda_{b},
\end{array}
$$


we have a unique connection in the third type bunch of connections:

$$
\begin{aligned}
& \stackrel{03}{\Gamma}_{\alpha \beta}^{a}=-\lambda_{\alpha \beta}^{a}-\mu_{\beta}^{a} \lambda_{\alpha}^{b} \lambda_{b}+\mu_{\beta}^{a} \lambda_{\alpha}-\Lambda_{\beta}^{a} \lambda_{\alpha}, \\
& 0{ }^{03} a b=-\lambda_{\alpha \beta}^{a b}+\Lambda_{\beta}^{a b} \lambda_{\alpha}^{c} \lambda_{c}-2 \Lambda_{\beta}^{a b} \lambda_{\alpha}, \\
& 0_{\alpha \beta}^{03}=-\lambda_{a \alpha}+\lambda_{a} \lambda_{\alpha}, \\
& \stackrel{03}{\Pi}_{a \alpha}^{b}=-\lambda_{a \alpha}^{b}-\delta_{a}^{b} \lambda_{c} \lambda_{\alpha}^{c}+2 \delta_{a}^{b} \lambda_{\alpha}, \\
& \stackrel{03}{L}_{\alpha \beta}=-\lambda_{\alpha \beta}+\lambda_{\alpha}^{a} \lambda_{a \beta}+\mu_{\beta}^{a} \lambda_{a} \lambda_{\alpha}-\lambda_{\alpha}^{a} \lambda_{a} \lambda_{\beta}, \\
& \stackrel{03}{\Pi}_{\alpha \beta}^{a}=-\chi_{\alpha \beta}^{a}-\Lambda_{\beta}^{b a} \lambda_{b} \lambda_{\alpha}+\lambda_{b \beta}^{a} \lambda_{\alpha}^{b}-\lambda_{\beta} \lambda_{\alpha}^{a}+\lambda_{\alpha}^{a} \lambda_{\beta}^{b} \lambda_{b} .
\end{aligned}
$$

Theorem. The analog of the strong Norden's normalization induces $(n-m)(m+$ 1) $\left(m^{2}+(n-m)^{2}\right)$-parameter third type bunch of group connections

$$
\stackrel{3}{\Gamma}=\left\{\Gamma_{1}, \stackrel{3}{\Gamma} \underset{\alpha \beta}{a}, \stackrel{3}{L_{\alpha \beta}^{a b}}, \stackrel{3}{L}_{a \alpha}, \stackrel{3}{\Pi}_{a \alpha}^{b}, \stackrel{3}{L}_{\alpha \beta}, \stackrel{3}{\Pi}_{\alpha \beta}^{a}\right\}
$$

in the fibering $G^{*}\left(\mathrm{Gr}^{*}(m, n)\right)$. The unique third type connection

$$
\stackrel{03}{\Gamma}=\left\{\stackrel{0}{\Gamma}_{b \alpha}^{a}, \stackrel{0}{L_{c \alpha}^{a b}}, \stackrel{0}{\Gamma} \underset{\beta \gamma}{\alpha}, \stackrel{0}{L_{\beta \gamma}^{\alpha a}}, \stackrel{03}{\Gamma} \underset{\alpha \beta}{a}, \stackrel{03}{L_{\alpha \beta}^{a b}}, \stackrel{03}{L_{a \alpha}}, \stackrel{03}{\Gamma_{a \alpha}^{b}}, \stackrel{03}{L_{\alpha \beta}}, \stackrel{03}{\Pi}_{\alpha \beta}^{a}\right\}
$$

is distinguished from this bunch.

\section{REFERENCES}

[1] O. Belova, "The second type connection in the fibering associated with the Grassmann-like manifold of centered planes," Differential geometry of manifolds of figures, vol. 38, pp. 6-12, 2007, in Russian.

[2] O. Belova, "The first type bunch of connections induced by an analog of Norden's normalization for the Grassmann-like manifold of centered planes," Ibid, vol. 41, pp. 13-17, 2010, in Russian.

[3] O. Belova, "Average connection in the fibering above Grassmann-like manifold of centered planes," Izvestia of the V.G. Belinsky PSPU, vol. 26, pp. 35-38, 2011, in Russian.

[4] A. P. Norden, Spaces with an affine connection. Moscow: Izdat. Nauka, 1976, in Russian.

Author's address

\section{Olga Belova}

Immanuel Kant Baltic Federal University, Department of Computer Safety, 14 A. Nevsky St., 236041 Kaliningrad, Russia

E-mail address: olgaobelova@mail.ru 